\title{
LETTER
}

\section{Hospital survey of point-of-care lung ultrasound use in the assessment of peri-operative and critically ill patients}

\author{
Kelvin Lam*1, David Canty², Colin Royse ${ }^{3}$ and Alistair Royse ${ }^{4}$
}

\section{Background}

Point-of-care lung ultrasonography is emerging as a valuable tool in critical care medicine. Advantages include rapid assessment of respiratory disease, which may be repeated and which does not expose patients or staff to ionizing radiation. This tool also avoids the need to transfer unstable patients to imaging departments and provides real-time assessment of organ function [1]. Certain applications, including detection of a pneumothorax or pleural effusion [2,3] and the improved safety of pleural drainage [4], are well established. Despite this, adoption by Australian clinicians into clinical practice has been slow. To determine the reasons for this, we surveyed 120 anesthesia, emergency, intensive care, and internal medicine consultants at the Royal Melbourne Hospital on their knowledge and practice of point-ofcare ultrasound. Approval for this study was obtained from the local Human Research Ethics Committee.

\section{Findings}

Fifty-eight clinicians out of 120 responded to the survey (48\%). Only 25 (43\%) clinicians reported knowledge of lung ultrasonography. Of these, 15 (26\%) perform lung ultrasound and the remaining 10 (17\%) do not, reportedly from lack of training. Of those performing lung ultrasound, only five (33\% of subgroup) had completed postgraduate ultrasonography training.

Most clinicians were aware that lung ultrasound can identify a pleural effusion (98\%) and pneumothorax (84\%), but fewer reported its ability to detect consolidation $(60 \%)$, atelectasis $(33 \%)$, and pulmonary edema (30\%). Sixteen (28\%) indicated that ultrasound equipment was not always available when required.

We conclude that under-utilization of lung ultrasound at this institution may be attributed to a combination of a lack of awareness, training, and equipment. In its finding of a paucity of formal training in ultrasonography among clinicians, this study concurs with a recent study by Lambert and colleagues [5]. Hospitals and training institutions need to provide greater availability of ultrasound equipment and training to facilitate greater adoption of lung ultrasound.

\section{Competing interests}

The authors declare that they have no competing interests.

\section{Acknowledgments}

The authors would like to thank the clinicians and administrative staff of the Royal Melbourne Hospital for their assistance with the survey.

\section{Author details}

'Department of Intensive Care, Royal Melbourne Hospital, Grattan Street, Parkville, Victoria, 3050, Australia. ${ }^{2}$ Department of Anaesthesia and Pain Management, Royal Melbourne Hospital, Grattan Street, Parkville, Victoria, 3050, Australia. ${ }^{3}$ Anaesthesia and Pain Management Unit, University of Melbourne, Grattan Street, Parkville, Victoria, 3050, Australia. ${ }^{4}$ Department of Cardiothoracic Surgery, Royal Melbourne Hospital, Grattan Street, Parkville, Victoria, 3050, Australia.

Published: 29 June 2012

\section{References}

1. Nolas M, Kot M, Mclean A, Lichtenstein D: Bedside lung ultrasound in the care of the critically ill. Curr Respir Med Rev 2010, 6:271-278.

2. Bouhemad B, Zhang M, Lu, Q, Rouby JJ: Clinical review: Bedside lung ultrasound in critical care practice. Crit Care 2007, 11:205-213.

3. Lichtenstein DA, Meziere GA: Relevance of lung ultrasound in the diagnosis of acute respiratory failure: the BLUE protocol. Chest 2008, 134:117-125.

4. Havelock T, Teoh R, Laws D, Gleeson F: Pleural procedures and thoracic ultrasound: British Thoracic Society Pleural Disease Guideline 2010. Thorax 2010, 65 (Suppl 2):61-76.

5. Lambert RL, Boker JR, Maffei FA: National survey of bedside ultrasound use in pediatric critical care. Pediatr Crit Care Med 2011, 12:655-659.

\section{doi:10.1186/cc11385}

Cite this article as: Lam K, et al:. Hospital survey of point-of-care lung ultrasound use in the assessment of peri-operative and critically ill patients. Critical Care 2012, 16:437.
*Correspondence: kelvinlam33@gmail.com

'Department of Intensive Care, Royal Melbourne Hospital, Grattan Street, Parkville, Victoria, 3050, Australia

Full list of author information is available at the end of the article 